\title{
Cobalt close-up
}

\section{David Lindsay and William Kerr remind us that where cobalt is concerned, good has triumphed over evil.}

C ompounds of cobalt have proved useful throughout history, and it remains an important element to this day, with applications in chemical synthesis. The name is thought to derive from the German kobold, meaning 'goblin' or 'evil sprite.' The difficulty in isolating cobalt from its ore - and the release of arsenic oxide that often accompanied the smelting process - were both attributed to the work of evil spirits by the miners who were tormented by this element. Undoubtedly, cobalt's relative scarcity also played a part - it makes up only 29 ppm of the earth's crust and is the thirtieth most abundant element on earth; the second rarest transition metal after scandium.

Cobalt was finally confirmed as a new element by Bergman in 1780, almost half a century after Brandt's isolation of the metal in 1735 . Cobalt's history goes back much further, however, as its ore was being used as a blue dye in the Middle East over four thousand years ago. Even today, almost $30 \%$ of cobalt produced is used in the ceramic and paint industries. Cobalt is also an essential trace element for humans, and is found at the centre of vitamin $\mathrm{B} 12$ and a range of other co-enzymes called cobalamins. Even though the body contains only $2-5 \mathrm{mg}$ of vitamin B12, its involvement in the production of red blood cells means it is vital to life. Vitamin $\mathrm{B} 12$ is also notable for its cobalt-carbon bond, making it the only known naturally occurring organometallic complex.

Similarly, many new developments in cobalt chemistry focus on organometallic compounds. In the field of catalysis, for example, cobalt has been found to mediate many of the cross-coupling reactions dominated for years by palladium and nickel. Furthermore, cobalt-based catalysts are also amongst the many systems being looked at for mediating the so-called oxygen evolution reaction (OER) - a fundamental step in the photochemically driven splitting of water into hydrogen and oxygen. The

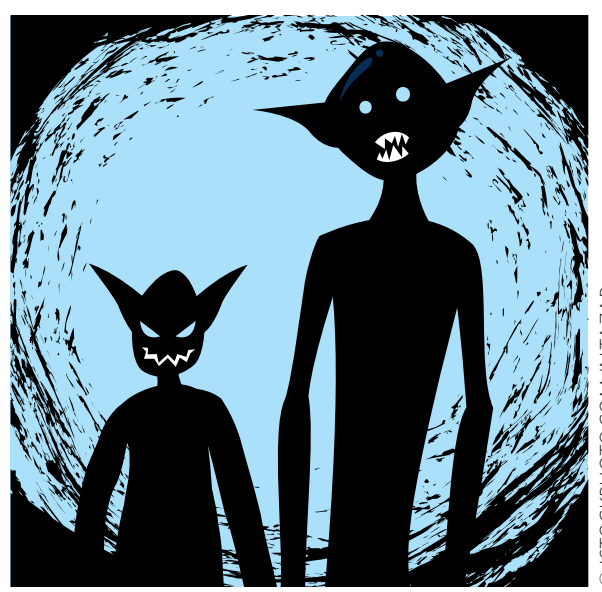

development of efficient technologies based on this process could ultimately lead to environmentally benign energy sources that harness the power of the Sun.

Historically, the most important application of organocobalt complexes in catalysis has been in the transformation of alkenes into aldehydes through a process known as hydroformylation. The classical catalyst for this reaction is the protonated derivative of the tetracarbonylcobaltate anion - $\mathrm{HCo}(\mathrm{CO})_{4}$. Although more selective catalysts, often rhodium-based, have been developed over the years, the cobalt system is still used for many applications.

Being a group 9 element, cobalt does not form a neutral monometallic carbonyl complex. Instead, it forms a dimer dicobalt octacarbonyl - with a bent cobaltcobalt bond. This dinuclear compound is also used as a hydroformylation catalyst, but another important reaction is one in which it reacts with alkynes - with loss of two molecules of carbon monoxide - to give alkyne-hexacarbonyl-dicobalt complexes. These air-stable and deep-red compounds can catalyse the cyclotrimerization of alkynes to make substituted benzenes, but their main preparative use is in the formation of substituted cyclopentenones.

The use of alkyne-cobalt complexes in such synthetically efficient and direct ring-forming processes was serendipitously discovered at the University of Strathclyde,
Scotland, in 1971. Following on from his pioneering work on the synthesis and reactivity of ferrocene, Peter L. Pauson turned his attention to the fundamental aspects of cobalt-mediated alkyne trimerization. Believing that sufficiently reactive alkenes may be able to react with alkyne-cobalt complexes in a similar fashion to alkynes, the appreciably strained alkene norbornene was investigated. The main organic products isolated from the reactions were cyclopentenones, formed from the combination of one unit each of the alkyne, alkene and carbon monoxide.

This accidental, yet extremely fortunate, discovery led to vigorous research efforts in the Strathclyde laboratories over subsequent years in an effort to establish the scope and limitations of this process for the onepot formation of selectively substituted cyclopentenones. Indeed, such is the importance of substituted five-memberedring ketones in organic chemistry, that many related metal-mediated processes for constructing such small ring systems have now evolved from these initial discoveries, and remain the focus of intense international research effort.

To (almost) all organic chemists the world over, this now renowned type of ring-building process is known as the Pauson-Khand reaction - that is, with the exception of one chemist: Pauson himself. The co-worker who discovered the cyclopentannulation process was Ihsan U. Khand, a postdoctoral researcher and exPhD student of Pauson's. Accordingly, and typical of his modesty and the generosity and support that he showed to his many students and colleagues, Pauson only ever referred to this reaction in spoken or written communications as 'the Khand reaction'.

\section{DAVID LINDSAY is at the Department} of Chemistry, University of Reading, Reading RG6 6AH, UK.

e-mail: d.lindsay@reading.ac.uk WILLIAM KERR is at the Department of Pure and Applied Chemistry, University of Strathclyde, Glasgow G1 1XL, UK. e-mail:w.kerr@strath.ac.uk
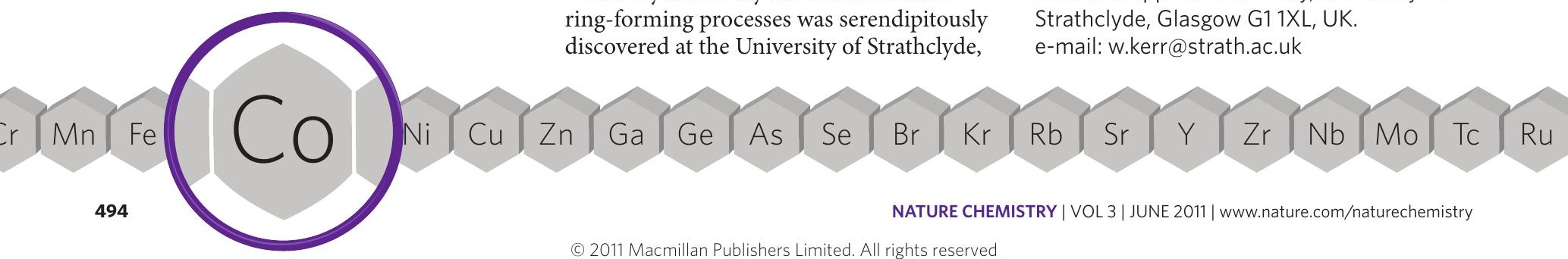